\title{
Comprehending isospin breaking effects of $X(3872)$ in a Friedrichs-model-like scheme
}

\author{
Zhi-Yong Zhou* \\ School of Physics, Southeast University, Nanjing 211189, People's Republic of China \\ Zhiguang Xiao ${ }^{\dagger}$ \\ Interdisciplinary Center for Theoretical Study, University of Science and Technology of China, \\ Hefei, Anhui 230026, China
}

(Received 20 November 2017; published 9 February 2018)

\begin{abstract}
Recently, we have shown that the $X(3872)$ state can be naturally generated as a bound state by incorporating the hadron interactions into the Godfrey-Isgur quark model using a Friedrichs-like model combined with the quark pair creation model, in which the wave function for the $X(3872)$ as a combination of the bare $c \bar{c}$ state and the continuum states can also be obtained. Under this scheme, we now investigate the isospin-breaking effect of $X(3872)$ in its decays to $J / \psi \pi^{+} \pi^{-}$and $J / \psi \pi^{+} \pi^{-} \pi^{0}$. By coupling its dominant continuum parts to $J / \psi \rho$ and $J / \psi \omega$ through the quark rearrangement process, one could obtain the reasonable ratio of $\mathcal{B}\left(X(3872) \rightarrow J / \psi \pi^{+} \pi^{-} \pi^{0}\right) / \mathcal{B}\left(X(3872) \rightarrow J / \psi \pi^{+} \pi^{-}\right) \simeq(0.58-0.92)$. It is also shown that the $\bar{D} D^{*}$ invariant mass distributions in the $B \rightarrow \bar{D} D^{*} K$ decays could be understood qualitatively at the same time. This scheme may provide more insight into the enigmatic nature of the $X(3872)$ state.
\end{abstract}

DOI: 10.1103/PhysRevD.97.034011

\section{INTRODUCTION}

The enigmatic $X(3872)$ state has been studied for more than a decade, after it was first discovered by the Belle Collaboration [1] and confirmed by the CDF, D0, and $B A B A R$ collaborations [2-4]. The mass of the $X(3872)$ is $3871.69 \pm 0.17 \mathrm{MeV}$ [5], almost degenerate with the $D^{* 0} \bar{D}^{0}$ threshold, which is the most intriguing feature. Its width is also very narrow, with an upper limit of $1.2 \mathrm{MeV}$. Its quantum number was determined to be $J^{P C}=$ $1^{++}$by the LHCb Collaboration [6], which is consistent with its radiative decay [7,8] and multipion transitions $[9,10]$. The negative result in searching for its charged partner in $B$ decays [11] implies that it should be an isoscalar state. Nevertheless, the dominant $\rho$ contribution of the dipion mass spectrum in $X(3872) \rightarrow J / \psi \pi^{+} \pi^{-}$[2] suggests a large isospin-breaking effect. Compared with its decay to $J / \psi \pi^{+} \pi^{-} \pi^{0}$ through the $I=0 \omega$ resonance, the ratio was measured to be

\footnotetext{
*zhouzhy@seu.edu.cn

${ }^{\dagger}$ Corresponding author. xiaozg@ustc.edu.cn

Published by the American Physical Society under the terms of the Creative Commons Attribution 4.0 International license. Further distribution of this work must maintain attribution to the author(s) and the published article's title, journal citation, and DOI. Funded by SCOAP.
}

$$
\frac{\Gamma\left(X(3872) \rightarrow J / \psi \pi^{+} \pi^{-} \pi^{0}\right)}{\Gamma\left(X(3872) \rightarrow J / \psi \pi^{+} \pi^{-}\right)}=1.0 \pm 0.4 \pm 0.3
$$

by the Belle Collaboration [12] and

$$
\frac{\Gamma\left(X(3872) \rightarrow J / \psi \pi^{+} \pi^{-} \pi^{0}\right)}{\Gamma\left(X(3872) \rightarrow J / \psi \pi^{+} \pi^{-}\right)}=0.8 \pm 0.3
$$

by the $B A B A R$ Collaboration [10]. These characteristics and other properties as discussed in the literature (see Refs. [13-17] for example) suggest an exotic nature of the $X(3872)$.

The proximity of the $X(3872)$ to the $D^{* 0} \bar{D}^{0}$ threshold leads to a direct interpretation of it as a hadronic molecular state. In fact, Törnqvist predicted a similar $D \bar{D}^{*}$ bound state around $3870 \mathrm{MeV}$ about ten years before the discovery of the $X(3872)$ [18], using a pion exchange model similar to the discussion of the deuteron in the $p n$ system, and named the state a "deuson." However, this picture may not explain the production cross section of $X(3872)$ in $p \bar{p}$ annihilation [19] and also cannot explain its large decay rate to $\gamma \psi^{\prime}$ $[20,21]$. This meson-exchange model was also extended to include other intermediate hadronic states like $\sigma, \rho$ and $\omega$ in Refs. [22-24]. The tetraquark model was also introduced to explain its existence and the other exotic states [25]. However, this explanation also meets the large $\gamma \psi^{\prime}$ decay rate problem. This bound state can also be reproduced by 
other models, such as the effective Lagrangian approach [26-29], chiral unitary calculation [30], screened potential model [24] and coupled-channel model [31]. It was also pointed out in Refs. [32,33] that $X(3872)$ could be a mixture of a $c \bar{c}$ and the molecule-like $D \bar{D}^{*}$ components which may provide a solution to both the production and the radiative decay problem. In our recent work [34], we discussed the first excited $P$-wave charmonium states using a Friedrichs-like model combined with the quark pair creation (QPC) model $[35,36]$ to incorporate the hadron interaction effect into the Godfrey-Isgur (GI) quark model [37]. We found that in the $2^{3} P_{1}$ channel the $X(3872)$ could be naturally generated as a bound state just below the $D^{0} \bar{D}^{0 *}$ threshold in this framework, while another resonance pole at around $3.934 \pm$ $0.040 i \mathrm{GeV}$ on the Riemann sheet attached to the physical region above the $\bar{D} D^{*}$ threshold is found to be generated from the bare $\chi_{c 1}(2 P)$, which might be related to the observed $X(3940)$ state in experiments. Compared to the above "deuson" picture, in this scheme, similar to Refs. [32,33], the $X(3872)$ is dynamically generated by the coupling of the bare $2^{3} P_{1} c \bar{c}$ state with the continuum, not from the pion exchange. The wave function of $X(3872)$ can also be explicitly written down as a linear combination of $c \bar{c}$ and the continuum states. With this information, more properties of the $X(3872)$ can be studied. In this paper, we will mainly focus on the isospin-breaking effect based on this result.

The ratio $\Gamma\left(X(3872) \rightarrow J / \psi \pi^{+} \pi^{-} \pi^{0}\right) / \Gamma(X(3872) \rightarrow$ $\left.J / \psi \pi^{+} \pi^{-}\right)$seems to imply a significant isospin-breaking effect, but it was first pointed out by Suzuki that it might be misleading [38]. Because the central mass value of $J / \psi \omega$ is about $7 \mathrm{MeV}$ higher than the $X(3872)$ mass, while the central value of $J / \psi \rho$ energy is lower than the $X(3872)$ mass, the $\pi^{+} \pi^{-} \pi^{0}$ in the $J / \psi \pi^{+} \pi^{-} \pi^{0}$ final state comes from the far tail of the $\omega$ resonance and the kinematical phase space of the isospin-conserved process is highly suppressed. Considering this kinematical constraint, the production amplitude ratio $\frac{A(X(3872) \rightarrow J / \psi \rho)}{A(X(3872 \rightarrow J / \psi \omega))}$ is estimated to be $0.27 \pm 0.02$ to produce a result comparable with experimental values. Meng and Chao addressed this problem by considering the rescattering effect in an effective Lagrangian method [39]. They treated the $X(3872)$ as an elementary field and introduced the effective interactions among the $X$ state, the pseudoscalar, and the vector mesons. By calculating the imaginary and real parts of the rescattering amplitudes, they found a consistent value of the ratio in their parameter space. Li and Zhu [40] investigated the probability in the framework of one-meson exchange by considering the S-D wave mixing and they determined the ratio of these two modes to be 0.42 . Gammermann and Oset [41] used the on-mass-shell Lippmann-Schwinger equation method and found a branch ratio of 1.4.

In the present paper, as stated above, we will discuss the isospin-breaking effect from the starting point of our previous paper [34]. The Friedrichs-like model is exactly solvable and the interactions between the bare $c \bar{c}$ and the Okubo-Zweig-Iizuka (OZI)-allowed continuum states are approximated by the QPC model using the wave functions for bare states from the GI model. This just provides a general scheme to make corrections to the well-accepted GI model by including the hadron interactions. The experimentally observed first excited $P$-wave charmonium states can be reasonably produced simultaneously. The wave functions for these states in terms of the bare $c \bar{c}$ and continuum states can also be explicitly written down. Once the wave function of the $X(3872)$ is explicitly given in this scheme, we are able to insert an interaction Hamiltonian between the $X(3872)$ wave function and the final states $J / \psi \omega$ or $J / \psi \rho$ and calculate the related transition amplitudes and the branching fraction. If the OZI-suppressed couplings are omitted, the $c \bar{c}$ contribution would be neglected and only the continuum components will contribute to the amplitude. The couplings between the final states and the continuum parts could then be described by the quark rearrangement model developed by Barnes and Swanson (BS model) [42]. The merit of this choice is that the BS model considers the spin-spin hyperfine, color Coulomb, and linear confinement interactions among the quarks between different mesons, which respects the same spirit as the GI model and no new parameter needs to be introduced. By a standard derivation of the representation of the transition amplitude and numerical calculations, our final result of $\frac{\Gamma\left(X(3872) \rightarrow J / \psi \pi^{+} \pi^{-} \pi^{0}\right)}{\Gamma\left(X(3872) \rightarrow J / \psi \pi^{+} \pi^{-}\right)}$turns out to be in good agreement with the experimental value. Our method may have some similarity with the widely used coupled-channel formalism such as in Refs. [43-48]. However, we are using the well-accepted GI wave function in the QPC model and the quark rearrangement model in our calculation, which provides a more solid theoretical basis and makes our result more convincing.

This paper is organized as follows. The theoretical foundations are introduced in Sec. II, which include the Friedrichs model, its extended scheme, and the quark rearrangement model by Barnes and Swanson. The numerical calculations of the isospin-breaking effect are given in Sec. III. Section IV contains our final conclusion and discussions.

\section{THEORETICAL MODEL}

In this section, we will briefly introduce the theoretical basis of our discussion which includes the main results of the Friedrichs model and its extended version, the quark rearrangement model by Barnes and Swanson, and the derivations of the transition amplitudes of $X(3872)$ to $J / \psi \omega$ or $J / \psi \rho$.

\section{A. The Friedrichs model}

In 1948, Friedrichs proposed an exactly solvable model to understand an unstable state [49]. The simplest form of 
the Friedrichs model includes a free Hamiltonian $H_{0}$ and an interaction part $V$. The free Hamiltonian $H_{0}$ has a bare continuous spectrum $\left[E_{t h}, \infty\right)$, and a discrete eigenvalue $E_{0}$ imbedded in this continuous spectrum $\left(E_{0}>E_{t h}\right)$. The interaction part describes the coupling between the bare continuous state and discrete states of $H_{0}$ so that the discrete state is dissolved in the continuous state and a resonance is produced.

In the energy representation, the free Hamiltonian can be expressed as

$$
H_{0}=E_{0}|0\rangle\left\langle 0\left|+\int_{E_{\text {th }}}^{\infty} E\right| E\right\rangle\langle E| \mathrm{d} E
$$

where $|0\rangle$ denotes the bare discrete state and $|E\rangle$ denotes the bare continuum state. The normalization conditions for the bare states are

$$
\langle 0 \mid 0\rangle=1, \quad\left\langle E \mid E^{\prime}\right\rangle=\delta\left(E-E^{\prime}\right), \quad\langle 0 \mid E\rangle=\langle E \mid 0\rangle=0 .
$$

The interaction part serves to couple the bare discrete state and the bare continuous state as

$$
V=\lambda \int_{E_{t h}}^{\infty}\left[f(E)|E\rangle\left\langle 0\left|+f^{*}(E)\right| 0\right\rangle\langle E|\right] \mathrm{d} E,
$$

where the $f(E)$ function denotes the coupling function and $\lambda$ denotes the coupling strength. This eigenvalue problem $H \Psi(x)=x \Psi(x)$, with the full Hamiltonian $H=H_{0}+V$, can be exactly solved and the final continuum state is

$$
\left|\Psi_{ \pm}(x)\right\rangle=|x\rangle+\lambda \frac{f^{*}(x)}{\eta^{ \pm}(x)}\left[|0\rangle+\lambda \int_{E_{t h}}^{\infty} \frac{f(E)}{x-E \pm i \epsilon}|E\rangle \mathrm{d} E\right]
$$

in which the resolvent $\eta^{ \pm}(x)$ is defined as

$$
\eta^{ \pm}(x)=x-E_{0}-\lambda^{2} \int_{E_{t h}}^{\infty} \frac{|f(E)|^{2}}{x-E \pm i \epsilon} \mathrm{d} E .
$$

The normalization is $\left\langle\Psi_{ \pm}(E) \mid \Psi_{ \pm}\left(E^{\prime}\right)\right\rangle=\delta\left(E-E^{\prime}\right)$ and the subscript \pm denotes the in states (+) and out states (-), respectively. The scattering $S$-matrix can also be obtained as

$$
S\left(E, E^{\prime}\right)=\delta\left(E-E^{\prime}\right)\left(1-2 \pi i \frac{\lambda f(E) f^{*}(E)}{\eta^{+}(E)}\right) .
$$

The $\eta^{ \pm}$functions can be analytically continued to the complex $z$ plane to be one complex function $\eta(z)$ for $z \in \mathbb{C}$ with its boundary values $\eta(x \pm i \epsilon)=\eta^{ \pm}(x)$ on the real axis. Since there is only one threshold for the continuum, there is only one cut for this function, and the $\eta(z)$ function is defined on a two-sheeted Riemann surface. The zero points for $\eta(z)=0$ will be the poles for the $S$-matrix.
The zero points of $\eta(z)$ or the poles of the $S$-matrix will represent the generalized complex-valued eigenstates (called the Gamow state) for the full Hamiltonian, which satisfy $H|z\rangle=z|z\rangle$ with $z \in \mathbb{C}$ as described in the riggedHilbert-space formulation of quantum mechanics developed by Bohm and Gadella [50,51]. By summing all the perturbation series, Prigogine and his collaborators also obtained a similar mathematical description [52]. In general, the different kinds of generalized eigenstates are summarized as follows:

1. Bound state:

The solution of $\eta(z)=0$ on the first-sheet real axis below $E_{t h}$ represents a bound state. Its wave function is written down as

$$
\left|z_{B}\right\rangle=N_{B}\left(|0\rangle+\lambda \int_{E_{t h}}^{\infty} \frac{f(E)}{z_{B}-E}|E\rangle \mathrm{d} E\right) .
$$

This solution has a finite norm and can be normalized as $\left\langle z_{B} \mid z_{B}\right\rangle=1$ where

$$
N_{B}=\left(\eta^{\prime}\left(z_{B}\right)\right)^{-1 / 2}=\left(1+\lambda^{2} \int \mathrm{d} E \frac{|f(E)|^{2}}{\left(z_{B}-E\right)^{2}}\right)^{-1 / 2} .
$$

Then, it is straightforward to define the so-called "elementariness" $Z$ and "compositeness" $X$ as

$$
Z=N_{B}^{2}, \quad X=\lambda^{2} N_{B}^{2} \int \mathrm{d} E \frac{|f(E)|^{2}}{\left(z_{B}-E\right)^{2}}
$$

similar to the ones in Weinberg's pioneering work in studying the compositeness of the deuteron [53]. The physical meanings of "elementariness" and "compositeness" are the probabilities of finding the bare discrete and the bare continuum states in the bound state respectively.

2. Virtual state:

The solution lying on the real axis of the second Riemann sheet below the threshold represents a virtual state. Its wave function is expressed as

$$
\left|z_{V}^{ \pm}\right\rangle=N_{V}\left(|0\rangle+\lambda \int_{E_{t h}}^{\infty} \frac{f(E)}{z_{V}^{ \pm}-E}|E\rangle \mathrm{d} E\right),
$$

where the superscripts \pm denote the two kinds of integration contours which are continued from the first sheet to the second sheet to enclose the virtual state pole from the upper side $(+)$ or the lower side $(-)$ of the first sheet cut [54]. Unlike the bound state, a virtual state does not have a well-defined norm as the usual state in the Hilbert space. Thus the compositeness and the elementariness for a virtual state cannot be mathematically rigorously defined. However, we can define a normalization such that $\left\langle z_{V}^{-} \mid z_{V}^{+}\right\rangle=1$, by choosing 
$N_{V}=\left(\eta^{\prime}\left(z_{V}\right)\right)^{-1 / 2}=\left(1+\lambda^{2} \int \mathrm{d} E \frac{|f(E)|^{2}}{\left(z_{V}-E\right)^{2}}\right)^{-1 / 2}$

One typical example is the virtual state in the singlet neutron-proton scattering, which serves to contribute to the unusually large scattering length.

3. Resonant state:

The real analyticity of the $\eta(z)$ function requires the solutions of $\eta(z)=0$ on the second complex energy plane to appear as a conjugate pair. This pair of poles of the $S$-matrix represents a resonance since it will be unstable for its finite imaginary part of the energy eigenvalue. The pole position on the lowerhalf energy plane of the second Riemann sheet is related to the mass $M$ and width $\Gamma$ as $z_{R}=M-i \frac{\Gamma}{2}$. The wave functions for them are written as

$$
\begin{aligned}
& \left|z_{R}\right\rangle=N_{R}\left(|0\rangle+\lambda \int_{E_{t h}}^{\infty} \mathrm{d} E \frac{f(E)}{\left[z_{R}-E\right]_{+}}|E\rangle\right), \\
& \left|z_{R}^{*}\right\rangle=N_{R}^{*}\left(|0\rangle+\lambda \int_{E_{t h}}^{\infty} \mathrm{d} E \frac{f^{*}(E)}{\left[z_{R}^{*}-E\right]_{-}}|E\rangle\right),
\end{aligned}
$$

where $z_{R}$ is on the lower half plane and $z_{R}^{*}$ is its complex conjugate. The $[\cdots]_{ \pm}$means the analytical continuations of the integrations from the upper or lower edge of the first sheet cut [54]. Similar to the virtual state, the wave function of a resonant state cannot be normalized as usual, and thus is not the normal state vector in the Hilbert space. However we can choose

$$
\begin{aligned}
N_{R} & =\left(\eta^{\prime+}\left(z_{R}\right)\right)^{-1 / 2} \\
& =\left(1+\lambda^{2} \int \mathrm{d} E \frac{|f(E)|^{2}}{\left[\left(z_{R}-E\right)_{+}\right]^{2}}\right)^{-1 / 2}
\end{aligned}
$$

to normalize the state as $\left\langle z_{R}^{*} \mid z_{R}\right\rangle=1$ and similarly for $\left|z_{R}^{*}\right\rangle$. The normalization factor $N_{R}$ will be complex in general and one might define "elementariness" and "compositeness" parameters as in Ref. [55], but their physical meanings are not clear because they are complex numbers too. However, some other physical approximate definitions proposed in Refs. [56,57] might be used to describe these quantities.

It is worth emphasizing that these discrete spectrum solutions may or may not be generated from the bare discrete state of the free Hamiltonian. If the state is generated from the bare discrete state, it will move back to the bare state as one decreases the coupling strength to zero. Besides, the discrete state could also be dynamically generated from the singularities of the form factor. In this case, if the coupling strength is tuned to be zero, the positions of this kind of state may move towards the singularity of the form factor on the unphysical sheets [54,58]. It is also worth mentioning that the Friedrichs model has several variants such as the Fano model [59], the Lee model [60], and the Anderson model [61] in other physics areas.

\section{B. The extended Friedrichs model and the QPC model}

In the original Friedrichs model, the states are labeled only by the energy quantum number which seems to be unrelated to the states in the three-dimensional space. In fact, after partial-wave decomposition of the three-dimensional states, a similar model in terms of the angular momentum eigenstate is reduced to a Friedrichs-like model [55]. Consider the coupling of a bare discrete state $|0, J M\rangle$ with a spin quantum number $J$ and the magnetic quantum number $M$ and the bare continuum momentum eigenstate $\left|\vec{p}, S, S_{z}\right\rangle$ with a total spin $S, z$ component $S_{z}$, and $\pm \vec{p}$ is the c.m. momentum for the two particles composing the continuum state for example. The discrete state denoted by the total Hamiltonian for fixed $J M$ can be recast into

$$
\begin{aligned}
H= & M_{0}|0\rangle\left\langle 0\left|+\sum_{L} \int \mathrm{d} E E\right| E, L\right\rangle\langle E, L| \\
& +\sum_{L} \int \mathrm{d} E f_{L}(E)|0\rangle\langle E, L|+\text { H.c. }
\end{aligned}
$$

in which $|0\rangle=|0 ; J M\rangle,|E, L\rangle=\sqrt{\mu p}|p ; J M ; L S\rangle$ where $\mu$ is the reduced mass of the two-particle state in its c.m. frame and $f(E)$ is the coupling form factor, as derived in Ref. [55]. This is just similar to the original Friedrichs model but with more continua and a similar exact solution can be obtained.

We can make a generalization by adding more discrete states and more continuum states, and the interactions between continuum states can also be introduced. The most general Hamiltonian with $D$ discrete states, $|i\rangle$ $(i=1, \ldots, D), \quad$ and $C$ continuum states, $\left|E_{j}, j\right\rangle$ $(j=1, \ldots, C)$, can be expressed as

$$
\begin{aligned}
H= & \sum_{i=1}^{D} M_{i}|0 ; i\rangle\left\langle 0 ; i\left|+\sum_{i=1}^{C} \int_{M_{i, t h}}^{\infty} \mathrm{d} E_{i} E_{i}\right| E_{i} ; i\right\rangle\left\langle E_{i} ; i\right| \\
& +\sum_{i_{2}, i_{1}} \int_{M_{i_{1}, t h}} \mathrm{~d} E^{\prime} \int_{M_{i_{2}, t h}} \mathrm{~d} E g_{i_{2}, i_{1}}\left(E^{\prime}, E\right)\left|E^{\prime} ; i_{2}\right\rangle\left\langle E ; i_{1}\right| \\
& + \text { H.c. } \\
& +\sum_{i=1}^{D} \sum_{j=1}^{C} \int_{M_{j, t h}} \mathrm{~d} E f_{i, j}(E)|0 ; i\rangle\langle E ; j|+\text { H.c. }
\end{aligned}
$$

where $f_{i, j}(E)$ is the form factor describing the interaction between the $i$ th discrete state and the $j$ th continuum state, and $g_{i j}$ describes the interaction between the $i$ th continuum 
and the $j$ th continuum. For general interactions $g_{i j}$, the model is not solvable, but if $g_{i j}\left(E^{\prime}, E\right)=v_{i j} f_{i}\left(E^{\prime}\right) f_{j}(E)$ and $f_{i j}=u_{i j} f_{j}(E)$, where $u_{i j}$ and $v_{i j}$ are constant, the model can also be exactly solved. See Ref. [55] for details.

In the present study, we only consider the case where only one discrete state is coupled with several continuum states without the interactions between the continuum states. The solutions differ from the ones previously described by simply adding more similar continuum integrals in Eqs. (7), (9), (12), and (14).

We will restrict our study to the properties of mesons. The next problem is to determine the interaction between the one-meson state and the two-meson continuum states, i.e. the coupling form factor in the Friedrichs model. A simple method is to use the QPC model $[35,36]$ to describe the interaction of one-meson and two-meson continuum states.

In this model, the meson coupling $A \rightarrow B C$ can be defined as the transition matrix element

$$
\langle B C|T| A\rangle=\delta^{3}\left(\overrightarrow{P_{f}}-\vec{P}_{i}\right) \mathcal{M}^{A B C}
$$

where the transition operator $T$ in the QPC model is defined as

$$
\begin{aligned}
T= & -3 \gamma \sum_{m}\langle 1 m 1-m \mid 00\rangle \int \mathrm{d}^{3} \overrightarrow{p_{3}} \mathrm{~d}^{3} \overrightarrow{p_{4}} \delta^{3}\left(\overrightarrow{p_{3}}+\overrightarrow{p_{4}}\right) \\
& \times \mathcal{Y}_{1}^{m}\left(\frac{\overrightarrow{p_{3}}-\overrightarrow{p_{4}}}{2}\right) \chi_{1-m}^{34} \phi_{0}^{34} \omega_{0}^{34} b_{3}^{\dagger}\left(\overrightarrow{p_{3}}\right) d_{4}^{\dagger}\left(\overrightarrow{p_{4}}\right),
\end{aligned}
$$

describing a quark-antiquark pair generated by the $b_{3}^{\dagger}$ and $d_{4}^{\dagger}$ creation operators from the vacuum. $\phi_{0}^{34}=(u \bar{u}+d \bar{d}+$ $s \bar{s}) / \sqrt{3}$ is the $S U(3)$ flavor wave function for the quarkantiquark pair. $\chi_{1-m}^{34}$ and $\omega_{0}^{34}$ are the spin wave function and the color wave function, respectively. $\mathcal{Y}_{1}^{m}$ is the solid harmonic function. $\gamma$ parametrizes the production strength of the quark-antiquark pair from the vacuum. The definition of the meson state here is different from the one in Ref. [62] as we omit the factor $\sqrt{2 E}$ to ensure the correct normalizations in the Friedrichs-like model,

$$
\begin{aligned}
\left|A\left(n,{ }^{2 S+1} L_{J, M}\right)(\vec{P})\right\rangle & \\
= & \sum_{M_{L}, M_{S}}\left\langle L M_{L} S M_{S} \mid J M\right\rangle \int \mathrm{d}^{3} p \psi_{n L M_{L}}(\vec{p}) \chi_{S M_{S}}^{12} \phi^{12} \omega^{12} \\
& \times\left|q_{1}\left(\frac{m_{1}}{m_{1}+m_{2}} \vec{P}+\vec{p}\right) \bar{q}_{2}\left(\frac{m_{2}}{m_{1}+m_{2}} \vec{P}-\vec{p}\right)\right\rangle .
\end{aligned}
$$

$\chi^{12}, \phi^{12}$ and $\omega^{12}$ are the spin wave function, flavor wave function and the color wave function, respectively. $p_{1}\left(p_{2}\right)$ and $m_{1}\left(m_{2}\right)$ are the momentum and mass of the quark (antiquark). $\vec{P}=\vec{p}_{1}+\vec{p}_{2}$ is the momentum of the c.m., and $\vec{p}=\frac{m_{2} \vec{p}_{1}-m_{1} \vec{p}_{2}}{m_{1}+m_{2}}$ is the relative momentum. $\psi_{n L M_{L}}$ is the wave function for the meson, with $n$ being the radial quantum number.

By the standard derivation one can obtain the amplitude $\mathcal{M}^{A B C}$ defined by Eq. (18) and the partial-wave amplitude $\mathcal{M}^{S L}(P(E))$ as in Ref. [36]. Then the form factor $f_{S L}$ which describes the interaction between $|A\rangle$ and $|B C\rangle$ in the Friedrichs model can be obtained as

$$
f_{S L}(E)=\sqrt{\mu P(E)} \mathcal{M}^{S L}(P(E)),
$$

where $P(E)=\sqrt{\frac{2 M_{B} M_{C}\left(E-M_{B}-M_{C}\right)}{M_{B}+M_{C}}}$ is the c.m. momentum, and $M_{B}$ and $M_{C}$ are the masses of mesons $B$ and $C$ respectively.

Now, the full Hamiltonian $H$ can be expressed as

$$
\begin{aligned}
H= & E_{0}|0\rangle\left\langle 0\left|+\sum_{n, S, L} \int_{E_{t h, n}}^{\infty} \mathrm{d} E E\right| E ; n, S L\right\rangle\langle E ; n, S L| \\
& +\sum_{n, S, L} \int_{E_{t h, n}}^{\infty} \mathrm{d} E f_{S L}^{n}(E)|0\rangle\langle E ; n, S L|+\text { H.c. }
\end{aligned}
$$

where $E_{0}$ denotes the bare mass of the discrete state, $n$ denotes the $n$th continuum state, $E_{t h, n}$ denotes the energy threshold of the $n$th continuum state, $S$ and $L$ are the total spin and the angular momentum of the continuum states, and $f_{S L}^{n}$ denote the coupling functions between the bare state and the $n$th continuum state with particular $S, L$ quantum numbers. The eigenvalue problem of the full Hamiltonian in Eq. (21) is exactly solvable as mentioned above. The final eigenvalues of bound states, virtual states or resonant states could be obtained by solving the resolvent function $\eta(z)=0$ on the complex energy plane where

$$
\eta(z)=z-E_{0}-\sum_{n} \int_{E_{t h, n}}^{\infty} \frac{\sum_{S, L}\left|f_{S L}^{n}(E)\right|^{2}}{z-E} \mathrm{~d} E .
$$

In Ref. [34], the $X(3872)$ and other first excited charmonium-like states could be generated based on the parameters and the wave functions of the GI model. $\gamma \sim 4.0$ is chosen such that the $X(3872)$ state emerges as a boundstate pole at around $3871.4 \mathrm{MeV}$, just below the $D^{0} \bar{D}^{0 *}$ threshold, which is not generated from the bare discrete state. This value is reasonable because the $\chi_{c 2}(2 P)$ and the $X(3872)$ in both channels match the experimental values simultaneously and a state around $3934 \mathrm{MeV}$ which is generated from $\chi_{c 1}(2 P)$ can also be assigned to the experimental observed $X(3940)$. This information provides the starting point for discussing the isospin-breaking property in the decays of the $X(3872)$ state. In this setup, the wave function of the $X(3872)$ is expressed explicitly as 


$$
\begin{aligned}
|X(3872)\rangle= & N_{B}\left(|c \bar{c}\rangle+\int_{M_{00 V}}^{\infty} \mathrm{d} E \sum_{S, L} \frac{f_{S L}^{00 V}(E)}{z_{X}-E}\left(\left|E ; D^{0} \bar{D}^{0 *}, S L\right\rangle+\left|E ; D^{0 *} \bar{D}^{0}, S L\right\rangle\right)\right. \\
& +\int_{M_{+-V}}^{\infty} \mathrm{d} E \sum_{S, L} \frac{f_{S L}^{+-V}(E)}{z_{X}-E}\left(\left|E ; D^{+} D^{-*}, S L\right\rangle+\left|E ; D^{+*} D^{-}, S L\right\rangle\right) \\
& \left.+\int_{M_{0 V 0 V}}^{\infty} \mathrm{d} E \sum_{S, L} \frac{f_{S L}^{0 V 0 V}(E)}{z_{X}-E}\left|E ; D^{0 *} \bar{D}^{0 *}, S L\right\rangle+\int_{M_{+V-V}}^{\infty} \mathrm{d} E \sum_{S, L} \frac{f_{S L}^{+V-V}(E)}{z_{X}-E}\left|E ; D^{+*} D^{-*}, S L\right\rangle\right),
\end{aligned}
$$

where $N_{B}=\eta^{\prime}\left(z_{X}\right)^{-1 / 2}$ is the normalization factor. The continuum states include $D^{0} \bar{D}^{0 *}, D^{0 *} \bar{D}^{0}, D^{ \pm} D^{\mp *}, D^{0 *} \bar{D}^{0 *}$, $D^{+*} D^{-*}$, and the corresponding thresholds and form factors are denoted using the subscripts and superscripts $00 V,+-V, 0 V 0 V$, and $+V-V$ respectively, with the charge-conjugate states sharing the same quantities. The $D_{s}^{ \pm} D_{s}^{* \mp}$ and $D_{s}^{* \pm} D_{s}^{* \mp}$ continuum states are not considered because it is much more difficult to produce the $s \bar{s}$ from the vacuum. The coupling form factor represents the interaction between the bare $c \bar{c}\left(2^{3} P_{1}\right)$ state from the GI model and the continuum states.

Since the process from $c \bar{c}$ to $J / \psi \rho$ and $J / \psi \omega$ are OZI suppressed, we do not include $J / \psi \rho$ and $J / \psi \omega$ in Eq. (17). However, the processes from $D \bar{D}^{*}$ and $D^{*} \bar{D}^{*}$ to $J / \psi \rho$ and $J / \psi \omega$ are not OZI suppressed. Furthermore, the relative ratio of finding $D \bar{D}^{*}$ in the $\mathrm{X}(3872)$ state is dominant over that of finding $c \bar{c}$ according to our previous study [34]. Thus, the contribution to the transition amplitude from $X(3872)$ to $J / \psi \rho$ and $J / \psi \omega$ is expected to come mainly from the continuum components, and the contribution from the $c \bar{c}$ component can be ignored.

Thus, the next task is to compute the transition amplitude from the continuum components to final states $|\rho J / \psi\rangle$ and $|\omega J / \psi\rangle$. This will be the main topic of the next section.

\section{The quark rearrangement model}

To evaluate the transition amplitude of the continuum component to the $J / \psi \omega$ and $J / \psi \rho$ states, a simple approach is to use the quark rearrangement method developed by Barnes and Swanson [42]. One of the merits of applying the BS model in our scheme is that the interaction in this model contains the one-gluon-exchange color Coulomb and spinspin interactions and linear scalar confinement terms, which are the nonrelativistic approximation of the GI model used here in determining the wave functions of the bare states. The interaction Hamiltonian of the BS model in the coordinate space is

$H_{I}=\sum_{i j}\left\{\frac{\alpha_{s}}{r_{i j}}-\frac{8 \pi \alpha_{s}}{3 m_{i} m_{j}} \vec{S}_{i} \cdot \vec{S}_{j} \delta\left(\vec{r}_{i j}\right)-\frac{3 b}{4} r_{i j}\right\} \mathbf{F}_{i} \cdot \mathbf{F}_{j}$

where the sum runs over all pairs $(i, j)$ of quarks and antiquarks in the hadrons. $\mathbf{F}_{i}$ denotes the color generator, $\alpha_{s}$ is the running coupling constant, and $b$ is the coupling strength of the linear potential. However, it is more convenient to make calculations in the momentum representation in accord with the extended Friedrichs model.

The main spirit of this model in calculating the mesonmeson scattering amplitude is to evaluate the lowest-order Born diagrams of the interchange processes of two constituent quarks, with the others being considered as spectators. The main formulas are summarized in the following and the details can be found in Refs. $[42,63]$. The momenta for the initial and final constituent quarks are denoted as $\vec{a}, \vec{b}$, $\vec{a}^{\prime}$, and $\vec{b}^{\prime}$ as shown in Fig. 1 and one can define the linear combinations $\vec{q}, \vec{p}_{1}$, and $\vec{p}_{2}$ as $\vec{q}=\vec{a}^{\prime}-\vec{a}=\vec{b}-\vec{b}^{\prime}$, $\vec{p}_{1}=\left(\vec{a}+\vec{a}^{\prime}\right) / 2$, and $\vec{p}_{2}=\left(\vec{b}+\vec{b}^{\prime}\right) / 2$. In the mesonmeson transition amplitude of $A B \rightarrow C D$, conservation of three-momentum implies that the matrix element is of the form

$$
\left\langle C D\left|H_{I}\right| A B\right\rangle=(2 \pi)^{-3} T_{f i} \delta(\vec{A}+\vec{B}-\vec{C}-\vec{D}) .
$$

The spin indices of $A, B, C$, and $D$ mesons are not written out explicitly. Four kinds of scattering diagrams, "capture ${ }_{1}$ " $\left(C_{1}\right)$, "capture 2 " $\left(C_{2}\right)$, "transfer ${ }_{1}$ " $\left(T_{1}\right)$, "transfer 2 " $\left(T_{1}\right)$ are considered as shown in Fig. 2, according to which pair of the constituents are involved in the interaction [42]. The $T$-matrix element of every diagram could be represented as the product of signature, flavor, color, spin, and space factors. The signature factor is $(-1)$ for all diagrams. The color factor is $(-4 / 9)$ for the two capture diagrams $\left(C_{1}\right.$ and $\left.C_{2}\right)$ and $(4 / 9)$ for the two transfer diagrams $\left(T_{1}\right.$ and $\left.T_{2}\right)$. The flavor factor is obtained by the overlap of the flavor wave functions of the initial and final states. The spin factors for $C_{1}, C_{2}, T_{1}$, and $T_{2}$ diagrams in different interaction terms are listed in Table I.

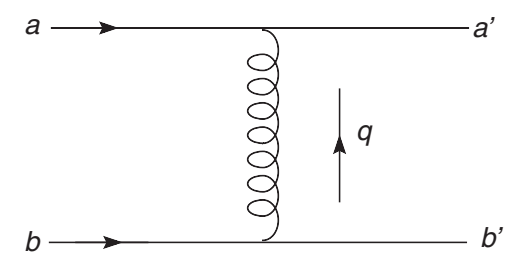

FIG. 1. Momentum definitions in the quark-quark transition amplitude. 

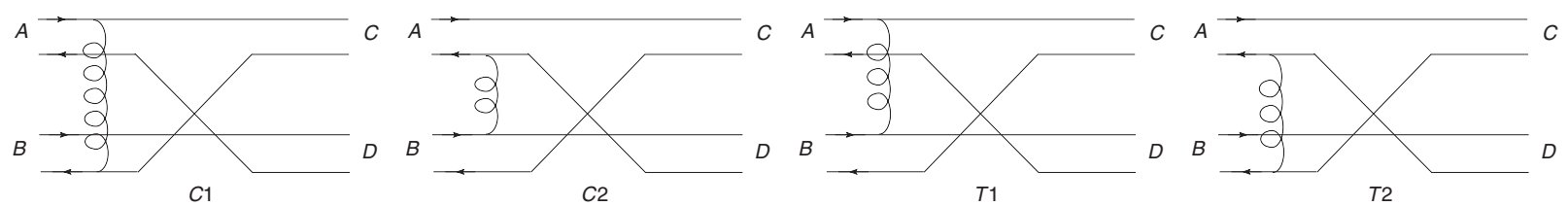

FIG. 2. The four-quark rearrangement diagrams of $A B \rightarrow C D$ meson-meson scatterings. The arrows represent the quark line directions.

The space overlap factor is obtained by

$$
\begin{aligned}
& I_{f i}^{\text {space }}(A B \rightarrow C D) \\
& \quad=\iint \mathrm{d}^{3} q \mathrm{~d}^{3} p \Phi_{C}^{*}\left(\vec{k}_{C}\right) \Phi_{D}^{*}\left(\vec{k}_{D}\right) T_{f i}^{\text {pot }}(\vec{q}) \Phi_{A}\left(\vec{k}_{A}\right) \Phi_{B}\left(\vec{k}_{B}\right)
\end{aligned}
$$

where $\vec{k}_{i}=\frac{m_{1} \vec{k}_{2}-m_{2} \vec{k}_{1}}{m_{1}+m_{2}}$ denotes the relative momentum of the quark-antiquark pair in the meson. $\vec{q}$ and $\vec{p}=\vec{p}_{1}$ are the only two independent variables, and the $T_{f i}^{\text {pot }}(\vec{q})$ refer to the interaction potential due to color Coulomb, spin-spin hyperfine and scalar confinement interactions in the momentum representation as

$T_{f i}^{\mathrm{pot}}(\vec{q})= \begin{cases}4 \pi \alpha_{s}(q) / q^{2}, & \text { color Coulomb } \\ -8 \pi \alpha_{s}(q) / 3 m_{i} m_{j}, & \text { spin-spin hyperfine } \\ 6 \pi b / q^{4}, & \text { linear confinement }\end{cases}$

where $m_{i}$ and $m_{j}$ are the constituent quark masses of the two interacting constituents. If the wave functions of the mesons are described as simple harmonic oscillator (SHO) wave functions, the integrations could be simplified and the analytical transition amplitudes of all four diagrams could be expressed explicitly in terms of confluent hypergeometric functions $[42,63]$. However, the meson wave functions of the GI model are represented in a basis of a large number of SHO wave functions, so the analytical expressions can hardly be obtained and we can only calculate the integrations numerically in a practical manner. In calculating the terms of the linear confinement interaction in the momentum space, the Hadamard regularization is used to regularize the divergent integrals to obtain the finite parts.

\section{Transition amplitude of $X(3872)$ to $J / \psi \rho$ and $J / \psi \omega$}

The BS model gives the transition amplitudes represented by the individual spins and the momenta of the initial and final mesons, and partial wave decomposition must be performed to obtain the matrix elements in term of the angular momentum eigenstates $\left\langle J / \psi \rho, S^{\prime} L^{\prime}\left|H_{I}\right| E\right.$; $\left.D^{0} \bar{D}^{0 *}, S L\right\rangle$. The $J / \psi \rho$ and $J / \psi \omega$ two-particle states are also decomposed in partial waves with total spin $S^{\prime}$ and angular momentum $L^{\prime}$. So, the transition amplitude of the $X(3872)$ to $J / \psi \rho$ with particular $S^{\prime}$ and $L^{\prime}$ is expressed as

$$
\begin{aligned}
M_{S^{\prime} L^{\prime}}(X(3872) \rightarrow J / \psi \rho)= & \left\langle J / \psi \rho, S^{\prime} L^{\prime}\left|H_{I}\right| X(3872)\right\rangle \\
= & N_{B}\left(\left\langle J / \psi \rho, S^{\prime} L^{\prime}\left|H_{I}\right| c \bar{c}\right\rangle\right. \\
& +\int \mathrm{d} E \sum_{S, L} \frac{f_{S L}^{00 V}(E)}{z_{X}-E}\left(\left\langle J / \psi \rho, S^{\prime} L^{\prime}\left|H_{I}\right| E ; D^{0} \bar{D}^{0 *}, S L\right\rangle+\left\langle J / \psi \rho, S^{\prime} L^{\prime}\left|H_{I}\right| E ; D^{0 *} \bar{D}^{0}, S L\right\rangle\right) \\
& +\int \mathrm{d} E \sum_{S, L} \frac{f_{S L}^{+-V}(E)}{z_{X}-E}\left(\left\langle J / \psi \rho, S^{\prime} L^{\prime}\left|H_{I}\right| E ; D^{+} D^{-*}, S L\right\rangle+\left\langle J / \psi \rho, S^{\prime} L^{\prime}\left|H_{I}\right| E ; D^{+*} D^{-}, S L\right\rangle\right) \\
& +\int \mathrm{d} E \sum_{S, L} \frac{f_{S L}^{0 V 0 V}(E)}{z_{X}-E}\left\langle J / \psi \rho, S^{\prime} L^{\prime}\left|H_{I}\right| E ; D^{0 *} \bar{D}^{0 *}, S L\right\rangle \\
& \left.+\int \mathrm{d} E \sum_{S, L} \frac{f_{S L}^{+V-V}(E)}{z_{X}-E}\left\langle J / \psi \rho, S^{\prime} L^{\prime}\left|H_{I}\right| E ; D^{+*} D^{-*}, S L\right\rangle\right)
\end{aligned}
$$

where the transition of $\left\langle J / \psi \rho\left|H_{I}\right| c \bar{c}\right\rangle$ will be omitted due to the OZI suppression, as mentioned above. A similar expression could be obtained for the $J / \psi \omega$ case.

Attention must be paid to the isospin difference of $\rho$ and $\omega$ whose flavor wave functions are $\rho=\frac{1}{\sqrt{2}}(u \bar{u}-d \bar{d})$ and $\omega=\frac{1}{\sqrt{2}}(u \bar{u}+d \bar{d})$ in ideal mixing, respectively, which lead to opposite signs in the flavor factors in the terms involving $d$ quark interchanges in the quark rearrangement model by Barnes and Swanson. 
TABLE I. Compilation of the spin factors for the $C_{1}, C_{2}, T_{1}$, and $T_{2}$ diagrams in the spin-spin hyperfine, color Coulomb, and linear potential terms with the total spin of two mesons being 1 [42].

\begin{tabular}{lccccccccc}
\hline \hline & \multicolumn{3}{c}{$(0,1) \rightarrow(1,1)$} & & \multicolumn{3}{c}{$(1,1) \rightarrow(1,1)$} \\
\cline { 2 - 4 } \cline { 5 - 8 }$\left(S_{A}, S_{B}\right) \rightarrow\left(S_{C}, S_{D}\right)$ & $C_{1}$ & $C_{2}$ & $T_{1}$ & $T_{2}$ & & $C_{1}$ & $C_{2}$ & $T_{1}$ & $T_{2}$ \\
\hline spin-spin & $\frac{1}{4 \sqrt{2}}$ & $\frac{1}{4 \sqrt{2}}$ & $-\frac{1}{4 \sqrt{2}}$ & $-\frac{1}{4 \sqrt{2}}$ & & 0 & 0 & $-\frac{1}{2}$ & $\frac{1}{2}$ \\
Coulomb & & & $\frac{1}{\sqrt{2}}$ & & & 0 & & \\
linear & & & $\frac{1}{\sqrt{2}}$ & & \multicolumn{2}{c}{0} & \\
\hline \hline
\end{tabular}

The observed final states of the $X(3872)$ decays are $J / \psi \pi^{+} \pi^{-}$through $\rho$ resonance and $J / \psi \pi^{+} \pi^{-} \pi^{0}$ through $\omega$ resonance. For simplicity, we describe the $\rho$ and $\omega$ resonances by their Breit-Wigner distribution functions [64], and then obtain

$$
\begin{aligned}
\Gamma(X & \left.\rightarrow J / \psi \rho\left(\pi^{+} \pi^{-}\right)\right) \\
& =\int_{2 m_{\pi}}^{m_{X}-m_{J / \psi}} \sum_{S, L} \frac{\left|M_{S, L}(X \rightarrow J / \psi \rho)\right|^{2} \Gamma_{\rho}}{\left(E-m_{\rho}\right)^{2}+\Gamma_{\rho}^{2} / 4} \mathrm{~d} E, \\
\Gamma(X & \left.\rightarrow J / \psi \omega\left(\pi^{+} \pi^{-} \pi^{0}\right)\right) \\
& =\int_{3 m_{\pi}}^{m_{X}-m_{J / \psi}} \sum_{S, L} \frac{\left|M_{S, L}(X \rightarrow J / \psi \omega)\right|^{2} \Gamma_{\omega}}{\left(E-m_{\omega}\right)^{2}+\Gamma_{\omega}^{2} / 4} \mathrm{~d} E,
\end{aligned}
$$

in which the lower limits of the integration are chosen at the experimental cutoffs as in Refs. [10,12].

\section{NUMERICAL RESULTS AND DISCUSSIONS}

In the numerical calculation, as in Ref. [34], we first reproduced the meson wave functions in the GI model [37] in a basis of a large number of SHOs, and applied them in the QPC model to obtain the coupling functions in the Friedrichs-like model. Then, the generalized energy eigenvalues of Gamow states and their wave functions were obtained by solving the Friedrichs-like model. The $X(3872)$ appears as a bound-state pole below the $\bar{D}^{0} D^{0 *}$ threshold and its wave function was obtained. Using the BS model and Eq. (28) we then calculated the transition amplitudes of the $X(3872)$ to the final states, and the branch ratio was thus obtained.

The parameters used in our calculation include all the parameters in the GI model, BS model and the $\gamma$ parameter in the QPC model. All the parameters in the GI model are fixed using the original GI values in order to obtain the meson wave functions including the bare $c \bar{c}$ state, the charmed mesons and $\rho, \omega$. The same parameters are used in the BS model without introducing any new parameters since these two models share similar interaction terms. The values of the two-particle thresholds in the Friedrichs-like model are chosen as their physical masses. Thus, all the parameters except for $\gamma$, symbolizing the quark pair production rate from the vacuum, are fixed at the values in the GI model [37].
The $\gamma$ parameter is chosen around 4.0 to produce a $X(3872)$ mass in the range $3.8710-3.8717 \mathrm{MeV}$ in order to be consistent with experimental values. There is also no cutoff parameter since the higher energy effects are suppressed in the integration by the form factor and the denominator, and thus the energies in the integration are integrated up to infinity. In this calculation, an explicit isospin-breaking effect is caused by considering the mass difference of $u$ and $d$ quarks with $\left(m_{u}+m_{d}\right) / 2=220 \mathrm{MeV}$ and $m_{d}-m_{u}=$ $5 \mathrm{MeV}$ [37]. The isospin-breaking effects can also be introduced into the production rate parameter through this mass difference by $\gamma=\frac{m_{u}+m_{d}}{2 m_{q}} \gamma_{0}$, where $m_{q}$ is the constituent quark mass of the produced pair [65].

GI wave functions of the bare meson states are approximately solved by expanding them in a basis of $30 \mathrm{SHO}$ wave functions. The wave function of the $X(3872)$ state as a bound state is also expressed as in Eq. (23). Then we can evaluate the partial-wave transition amplitudes of neutral or charged $D \bar{D}^{*}$ to $J / \psi \rho$ or $J / \psi \omega$. In the BS quark rearrangement model, the spin and angular momentum are conserved separately, so the partial $L$ wave of the $J / \psi \rho, \omega$ states can only couple to the same partial $L$-wave part in the $D \bar{D}^{*}$ continuum term. Since the vector-vector $D^{*} \bar{D}^{*}$ could only have the $D$-wave continuum in the QPC coupling, they will only contribute to the $D$-wave $J / \psi \rho$ or $J / \psi \omega$. The magnitudes of the $D$-wave scattering amplitudes of $D^{*} \bar{D}^{*}$ to $J / \psi \rho$ or $J / \psi \omega$ are highly suppressed and they are several orders of magnitude lower than that of $S$-wave $\bar{D} D^{*}$ amplitudes. So, the main contributions are from the $D \bar{D}^{*}$ continuum parts. Furthermore, the $D^{*} D^{*}$ continuum component in $X(3872)$ is very small. The ratio of "elementariness" and "compositeness" of the different components in the $X(3872)$ is about $Z_{c \bar{c}}: X_{\bar{D}^{0} D^{0 *}}: X_{D^{+} D^{-*}}: X_{\bar{D}^{*} D^{*}}=$ $1:(2.67-8.85):(0.45-0.46): 0.04$ according to Eq. (11) as $\gamma$ is tuned to obtain the $X(3872)$ mass in the range $3.8710-3.8717 \mathrm{MeV}$. It is natural that the compositeness is sensitive to the closeness of the pole to the $\bar{D}^{0} D^{0 *}$ threshold due to the denominator in the integrand in Eq. (11). This denominator will amplify the form factor near the threshold, and thus the integral is sensitive to the near-threshold behaviors of the coupling form factor $f_{S L}(E)$. Since the form factor is calculated using the wave function, different choices of the wave functions may also affect the behavior of the form factor near the threshold. Here, we choose the well-accepted GI wave functions as the input, which is more accurate than just using a phenomenological monopole form factor like in Refs. [39,46].

The isospin-breaking effect is caused by the mass difference of the constituent $u$ and $d$ quarks. This leads to two consequences in the calculation. First, it causes the mass difference of the $\bar{D}^{0} D^{* 0}$ and $\bar{D}^{+} D^{*-}$. If the isospin symmetry is respected, the $\bar{D}^{0} D^{* 0}$ and $\bar{D}^{+} D^{*-}$ will be degenerate and their contributions to the scattering amplitude to $J / \psi \rho$ from corresponding terms in Eq. (28) will be 

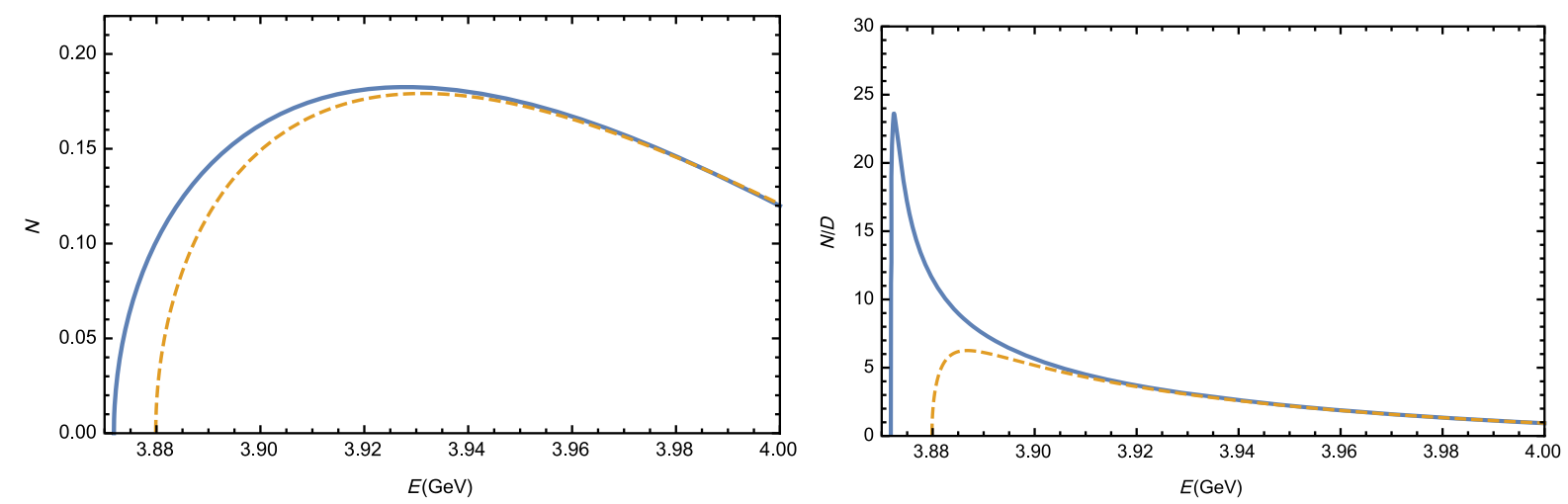

FIG. 3. Comparison of the integrand of Eq. (28) for charged and neutral channels without or with the denominators $(N$ or $N / D)$ respectively. The solid line represents the neutral $\bar{D} D^{*}$ channel and the dashed one the charged channel. The left panel shows the contributions of the numerator only and the right panel shows the whole integrand with the denominators.

totally canceled but not in the scattering amplitude to $J / \psi \omega$. Now that the threshold for $\bar{D}^{0} D^{* 0}$ is lower than $\bar{D}^{+} D^{*-}$ by about $8 \mathrm{MeV}, \bar{D}^{0} D^{* 0}$ will give a nonzero contribution to the amplitude to $J / \psi \rho$ below the $\bar{D}^{+} D^{*-}$ threshold. Above the $\bar{D}^{+} D^{*-}$ threshold, in general, the nearer it is to the threshold, the larger the difference between the two amplitudes due to the mass difference. In addition, the $\gamma$ symbolizing the quark pair creation strength from the vacuum also depends on the quark masses, which also leads to a smaller coupling to the $\bar{D}^{+} D^{-*}$ continuum than to the $\bar{D}^{0} D^{0 *}$ continuum. It is natural that the heavier components are more difficult to produce. These two effects caused by the explicit isospinbreaking of $u$ and $d$ quark masses are still quite tiny since $m_{d}-m_{u}$ is about $5 \mathrm{MeV}$ in the GI model. However, it is greatly amplified by the denominators of $\frac{1}{z_{X}-E}$ in Eq. (28) because the mass of $X(3872)$ is very close to the $\bar{D}^{0} D^{* 0}$ threshold. To demonstrate this mechanism clearly, we plot the numerator part in the integrand and the whole integrand of neutral and charged $\bar{D} D^{*}$ terms in Eq. (28), respectively, for the amplitude to $J / \psi \rho$ in Fig. 3 for comparison. The left panel shows the absolute values of the numerators of neutral (solid) and charged (dashed) $\bar{D} D^{*}$ contributions in Eq. (28), which are slightly different. However, the whole integrand with the $\frac{1}{z_{x}-E}$ denominator shown in the right panel is totally different. We see that near the threshold the differences between the two integrands are greatly enhanced by the denominator and hence give a sizable contribution to the amplitude.

After the amplitudes are calculated by integrating up to infinity in Eq. (28), one could obtain the relative ratio of the transition amplitudes, $\frac{M(X \rightarrow J / \psi \omega) / \sqrt{P_{J / \psi \omega}}}{M(X \rightarrow J / \psi \rho) / \sqrt{P_{J / \psi \rho}}}=3.24 \sim 2.43$, with the $X(3872)$ mass ranging from $3871.0-3871.7 \mathrm{MeV}$, which is consistent with the expectation of Suzuki [38] to produce a reasonable isospin breaking. In principle, to obtain the decay widths using Eq. (29), the $\rho, \omega$ masses in the scattering amplitudes of $\bar{D} D^{*} \rightarrow J / \psi \rho, \omega$ should be regarded as variables. However, since the numerical calculation of the scattering amplitudes involve sixdimensional integration, the integration would be difficult and costs too much computer time. We have checked that the numerical values of the scattering amplitudes change very slowly when the masses of $\rho, \omega$ change. Thus, in a practical manner, we did not calculate the integration directly but approximately calculated the scattering amplitudes with the $\rho, \omega$ masses fixed at their Particle Data Group (PDG) central values and product the phase space kinematic factors. If we cut off the $\pi^{+} \pi^{-}$invariant mass at $0.45 \mathrm{GeV}$ and the $\pi^{+} \pi^{-} \pi^{0}$ invariant mass at $0.75 \mathrm{GeV}$ as chosen by the Belle and $B A B A R$ collaborations $[10,12]$, the kinematic space ratio will be

$$
\frac{\int_{3 m_{\pi}}^{m_{X}-m_{J / \psi}} \frac{\left|P_{J / \psi \omega}\right| \Gamma_{\omega}}{\left(E-m_{\omega}\right)^{2}+\Gamma_{\omega}^{2} / 4} \mathrm{~d} E}{\int_{2 m_{\pi}}^{m_{X}-m_{J / \psi}} \frac{\left|P_{J / \psi \psi}\right| \Gamma_{\rho}}{\left(E-m_{\rho}\right)^{2}+\Gamma_{\rho}^{2} / 4} \mathrm{~d} E}=0.088 \sim 0.098
$$

as the mass of $X(3872)$ ranges from $3871.0-3871.7 \mathrm{MeV}$ and the branch fraction will be

$$
\begin{aligned}
\frac{\Gamma_{J / \psi \pi^{+} \pi^{-} \pi^{0}}}{\Gamma_{J / \psi \pi^{+} \pi^{-}}} & =(3.24 \sim 2.43)^{2} \times(0.088 \sim 0.098) \\
& =0.92 \sim 0.58 .
\end{aligned}
$$

This numerical results are consistent with the measured ratio $1.0 \pm 0.4 \pm 0.3$ by Belle [12] and $0.8 \pm 0.3$ by $B A B A R$ [10]. If the lower limits are chosen at the $2 m_{\pi}$ and $3 m_{\pi}$ physical masses respectively, the ratio of kinematic factors will be $0.161-0.169$ and

$$
\begin{aligned}
\frac{\Gamma_{J / \psi \pi^{+} \pi^{-} \pi^{0}}}{\Gamma_{J / \psi \pi^{+} \pi^{-}}} & =(3.24 \sim 2.43)^{2} \times(0.161 \sim 0.169) \\
& =1.69 \sim 0.99 .
\end{aligned}
$$

For completeness, we also list here the estimated decay widths: $\Gamma_{J / \psi\left(\rho \rightarrow \pi^{+} \pi^{-}\right)}$is $1 \sim 3 \mathrm{keV}$ and $\Gamma_{J / \psi\left(\omega \rightarrow \pi^{+} \pi^{-} \pi^{0}\right)}$ $1-5 \mathrm{keV}$. The present experiments have not provided much 

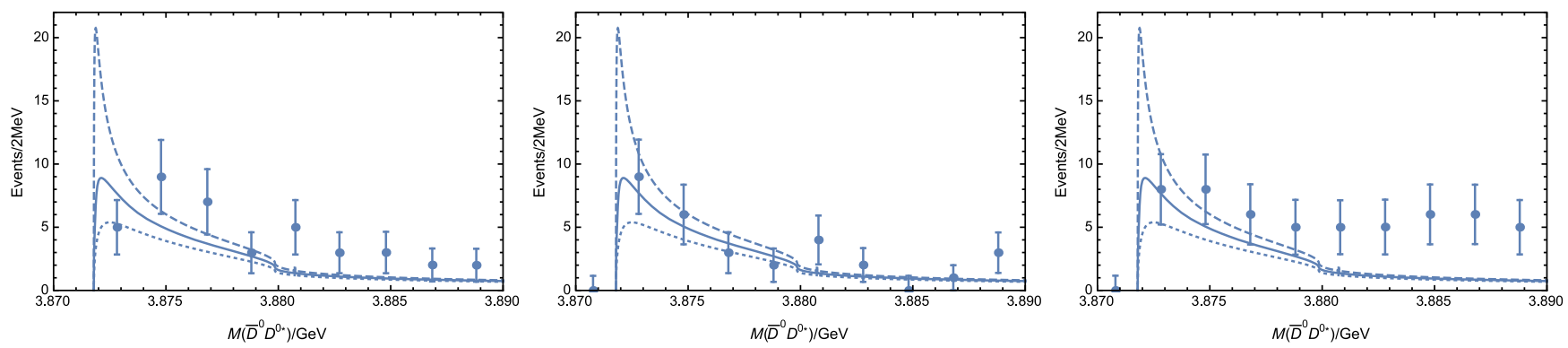

FIG. 4. The experimental $\bar{D}^{0} D^{0 *}$ mass distributions in $B \rightarrow X\left(\bar{D}^{0} D^{0 *}\right) K$ decays measured by $B A B A R$ (left) [68] and Belle (middle and right) [67] compared with $r\left|T\left(\bar{D}^{0} D^{0 *} \rightarrow \bar{D}^{0} D^{0 *}\right)\right|^{2}$. phase space, where $r$ is a rescaling factor chosen by hand. The dotted, solid, and dashed lines represent the curves with the $X(3872)$ mass at $3.8710,3.8714$, and $3.8717 \mathrm{GeV}$, respectively.

information on these two decay widths. According to the PDG [5], the only useful information is the upper limit of the $X(3872)$ total decay width $\Gamma_{X(3872)}<1.2 \mathrm{MeV}$, and the branching fraction $\frac{\Gamma_{J / \psi\left(\rho \rightarrow \pi^{+} \pi^{-}\right)}}{\Gamma_{X(3872)}}>0.026$. In fact, the absolute value for the decay width in our calculation depends much on the parametrization of the strong coupling constant. In the GI model it is parametrized as $\alpha_{s}\left(q^{2}\right)=0.25 \exp \left(-q^{2}\right)+$ $0.15 \exp \left(-q^{2} / 10\right)+0.20 \exp \left(-q^{2} / 1000\right)$ to avoid the divergence near $\Lambda_{\mathrm{QCD}}$, which is what we used in our calculation in the BS model. This parametrization respects the large-momentum perturbative behavior and smoothes the low-momentum region by assuming $\alpha_{s}\left(q^{2}=0\right)=0.6$ in the confinement region. However, in BS's original model, they used just a constant within $0.5-0.6[63,66]$. If we also use this constant in our calculation, the obtained decay width will be enhanced by about an order of magnitude with the relative ratio almost unchanged.

As a byproduct, this scheme can also provide a qualitative interpretation of the $\bar{D}^{0} D^{0 *}$ mass distribution in $B \rightarrow$ $\bar{D}^{0} D^{0 *} K$ processes by the Belle and $B A B A R$ collaborations $[67,68]$. Since the multichannel scattering amplitudes could be obtained similar to Eq. (8) as in Ref. [55], the $T$-matrix of $\bar{D}^{0} D^{0 *}$ scatterings could be obtained as

$$
T_{S L}\left(\bar{D}^{0} D^{0 *} \rightarrow \bar{D}^{0} D^{0 *}\right)=\frac{\left|f_{S L}^{\bar{D}^{0} D^{0 *}}(E)\right|^{2}}{\eta^{+}(E)}
$$

If the weak interaction vertex in $B \rightarrow X\left(\bar{D}^{0} D^{0 *}\right) K$ decays is supposed to be a smooth mildly changing factor and could be simulated by a constant, the mass distribution of the $\bar{D}^{0} D^{0 *}$ final states is proportional to $\left|T\left(\bar{D}^{0} D^{0 *} \rightarrow \bar{D}^{0} D^{0 *}\right)\right|^{2}$ and the phase space factors. A qualitative agreement could be found between the calculations and the experimental data up to a rescaling factor, as shown in Fig. 4, which is similar to the result in the near-threshold parametrization method [69]. The dotted, solid, and dashed lines in Fig. 4 represent the $X(3872)$ mass at $3.8710,3.8714$, and $3.8717 \mathrm{GeV}$, respectively, up to a rescaling factor.

\section{SUMMARY}

In this paper, we performed a calculation on the branching ratio of the $X(3872)$ transition to $J / \psi \pi^{+} \pi^{-} \pi^{0}$ and $J / \psi \pi^{+} \pi^{-}$ based on a Friedrichs-like scheme combined with the quark rearrangement model and QPC model. In our previous work [34], the first excited $P$-wave charmonium state spectrum was reproduced using the same Friedrichs scheme combined with the QPC model using the GI bare spectrum as input, and the results were consistent with experimental values, which demonstrated the reasonability of this scheme. In fact, this scheme provides a general way to incorporate the hadron interactions into the GI quark model. The $X(3872)$ is dynamically generated as a bound state just below the $D^{* 0} \bar{D}^{0}$, composed of a dominant $\bar{D}^{0} D^{0 *}$ continuum component of about $64-85 \%$, a $c \bar{c}$ component of about $10-24 \%$, and other continua. The $X(3872)$ wave function can be explicitly expressed as the linear combination of the $c \bar{c}$ states and the continuum states. Based on this information, in the present paper we studied the isospin-breaking effect within this framework. Since the $X(3872)$ is mostly composed of continuum and the $c \bar{c}$ contribution is also suppressed by the OZI rule, we could only consider the continuum contributions to the decay amplitude. In the spirit of the quark rearrangement model, by considering the spin-spin, color Coulomb, and linear potential interactions among the quarks in different mesons, one can obtain the transition amplitudes for $X(3872)$ to $J / \psi \omega$ and $J / \psi \rho$, using the wave function obtained from the Friedrichs scheme. By taking into account the mass difference of the final states, we obtained the numerical result $\frac{\Gamma_{J / \psi \pi^{+} \pi^{-} \pi^{0}}}{\Gamma_{J / \psi \pi^{+} \pi^{-}}}=$ $0.92-0.58$ as the $X(3872)$ mass changes from 3871.0 to $3871.7 \mathrm{MeV}$, which is comparable to the experimental value. We noticed that to provide a reasonable magnitude for the isospin-breaking amplitude, the proximity of the $X(3872)$ position to the $D^{0} D^{0 *}$ plays an important role. It greatly amplifies the amplitude to $D^{0} \bar{D}^{0 *}$ near the threshold which causes a large isospin-breaking effect in the amplitude as shown in Fig. 3. This amplification effect is also present in the integration in the compositeness calculation. Thus, the precise near-threshold behavior of the form factor is 
important for this result to be solid. Since our calculations are based on GI wave functions, which supposedly are the de facto standard in the literature, they presumably provide a more precise form factor which makes our results more solid and convincing.

In this calculation, because the interaction Hamiltonian between the quarks of different mesons in the quark rearrangement model is similar to GI's quark potential model, there are no new parameters introduced in the calculation. All the model parameters except the quark production rate from the vacuum, $\gamma$, are fixed at the wellaccepted GI model. The $\gamma$ parameter is also chosen such that the $X(3872)$ is around the experimental mass and all the other observed first excited $P$-wave states are reproduced well as in Ref. [34]. One of the merits of using our scheme is that the high-energy contribution in the integration in calculating the decay amplitudes is naturally suppressed by the form factor obtained from the QPC model based on the wave function from GI's relativized quark model. Thus, unlike in Refs. [38,39] where the result was cutoff dependent, here no cutoff was introduced and thus the result is more robust.

\section{ACKNOWLEDGMENTS}

Helpful discussions with Dian-Yong Chen, HaiQing Zhou, Ce Meng, and Xiao-Hai Liu are appreciated. Z.X. is supported by the China National Natural Science Foundation under contracts No. 11105138, No. 11575177 and No. 11235010. Z.Z is supported by the Natural Science Foundation of Jiangsu Province of China under contract No. BK20171349.
[1] S. K. Choi et al. (Belle Collaboration), Phys. Rev. Lett. 91, 262001 (2003).

[2] D. Acosta et al. (CDF Collaboration), Phys. Rev. Lett. 93, 072001 (2004).

[3] V. M. Abazov et al. (D0 Collaboration), Phys. Rev. Lett. 93, 162002 (2004).

[4] B. Aubert et al. (BABAR Collaboration), Phys. Rev. D 71, 071103 (2005).

[5] C. Patrignani et al., Chin. Phys. C 40, 100001 (2016).

[6] R. Aaij et al. (LHCb Collaboration), Phys. Rev. Lett. 110, 222001 (2013).

[7] V. Bhardwaj et al. (Belle Collaboration), Phys. Rev. Lett. 107, 091803 (2011).

[8] B. Aubert et al. (BABAR Collaboration), Phys. Rev. D 74, 071101 (2006).

[9] A. Abulencia et al. (CDF Collaboration), Phys. Rev. Lett. 96, 102002 (2006).

[10] P. del Amo Sanchez et al. (BABAR Collaboration), Phys. Rev. D 82, 011101 (2010).

[11] B. Aubert et al. (BABAR Collaboration), Phys. Rev. D 71, 031501 (2005).

[12] K. Abe et al. (Belle Collaboration), in Lepton and Photon Interactions at High Energies: Proceedings of the XXII International Symposium, Uppsala, Sweden, June 30-July 5, 2005, edited by R. Brenner, C. P. de los Heros, and J. Rathsman (World Scientific, Singapore, 2005).

[13] F.-K. Guo, C. Hanhart, Ulf-G. Meiner, Q. Wang, Q. Zhao, and B.-S. Zou, arXiv:1705.00141 [Rev. Mod. Phys. (to be published)].

[14] H.-X. Chen, W. Chen, X. Liu, and S.-L. Zhu, Phys. Rep. 639, 1 (2016).

[15] A. Esposito, A. Pilloni, and A. D. Polosa, Phys. Rep. 668, 1 (2017).

[16] R. F. Lebed, R. E. Mitchell, and E. S. Swanson, Prog. Part. Nucl. Phys. 93, 143 (2017).
[17] S. L. Olsen, T. Skwarnicki, and D. Zieminska, arXiv: 1708.04012 [Rev. Mod. Phys. (to be published)].

[18] N. A. Tornqvist, Z. Phys. C 61, 525 (1994).

[19] C. Bignamini, B. Grinstein, F. Piccinini, A. D. Polosa, and C. Sabelli, Phys. Rev. Lett. 103, 162001 (2009).

[20] E. S. Swanson, Phys. Lett. B 588, 189 (2004).

[21] Y. Dong, A. Faessler, T. Gutsche, and V. E. Lyubovitskij, J. Phys. G 38, 015001 (2011).

[22] Y.-R. Liu, X. Liu, W.-Z. Deng, and S.-L. Zhu, Eur. Phys. J. C 56, 63 (2008).

[23] X. Liu, Z.-G. Luo, Y.-R. Liu, and S.-L. Zhu, Eur. Phys. J. C 61, 411 (2009).

[24] B.-Q. Li and K.-T. Chao, Phys. Rev. D 79, 094004 (2009).

[25] L. Maiani, F. Piccinini, A. D. Polosa, and V. Riquer, Phys. Rev. D 71, 014028 (2005).

[26] M. T. AlFiky, F. Gabbiani, and A. A. Petrov, Phys. Lett. B 640, 238 (2006).

[27] S. Fleming, M. Kusunoki, T. Mehen, and U. van Kolck, Phys. Rev. D 76, 034006 (2007).

[28] N. N. Achasov and E. V. Rogozina, Mod. Phys. Lett. A 30, 1550181 (2015).

[29] N. N. Achasov and E. V. Rogozina, J. Univ. Sci. Tech. China 46, 574 (2016).

[30] P. Wang and X. G. Wang, Phys. Rev. Lett. 111, 042002 (2013).

[31] Z.-Y. Zhou and Z. Xiao, Eur. Phys. J. A 50, 165 (2014).

[32] E. Braaten and M. Lu, Phys. Rev. D 76, 094028 (2007).

[33] S. Coito, G. Rupp, and E. van Beveren, Eur. Phys. J. C 73, 2351 (2013).

[34] Z.-Y. Zhou and Z. Xiao, Phys. Rev. D 96, 054031 (2017); 96, 099905(E) (2017).

[35] L. Micu, Nucl. Phys. B10, 521 (1969).

[36] H. G. Blundell and S. Godfrey, Phys. Rev. D 53, 3700 (1996).

[37] S. Godfrey and N. Isgur, Phys. Rev. D 32, 189 (1985).

[38] M. Suzuki, Phys. Rev. D 72, 114013 (2005). 
[39] C. Meng and K.-T. Chao, Phys. Rev. D 75, 114002 (2007).

[40] N. Li and S.-L. Zhu, Phys. Rev. D 86, 074022 (2012).

[41] D. Gamermann and E. Oset, Phys. Rev. D 80, 014003 (2009).

[42] T. Barnes and E. S. Swanson, Phys. Rev. D 46, 131 (1992).

[43] E. Eichten, K. Gottfried, T. Kinoshita, K. D. Lane, and T.-M. Yan, Phys. Rev. D 17, 3090 (1978); 21, 313(E) (1980).

[44] Y. S. Kalashnikova, Phys. Rev. D 72, 034010 (2005).

[45] P. G. Ortega, J. Segovia, D. R. Entem, and F. Fernandez, Phys. Rev. D 81, 054023 (2010).

[46] M. Takizawa and S. Takeuchi, Prog. Theor. Exp. Phys. 2013, 093D01 (2013).

[47] X. Cao and H. Lenske, arXiv:1408.5600.

[48] P. G. Ortega and E. Ruiz Arriola, in Proceedings of the 17th International Conference on Hadron Spectroscopy and Structure (Hadron 2017) Salamanca, Spain, September 25-29, 2017 (to be published) [arXiv:1711.10193].

[49] K. O. Friedrichs, Commun. Pure Appl. Math. 1, 361 (1948).

[50] A. Bohm and M. Gadella, Dirac Kets, Gamow Vectors and Gel'fand Triplets, edited by A. Bohm and J. D. Dollard (Springer, New York, 1989).

[51] O. Civitarese and M. Gadella, Phys. Rep. 396, 41 (2004).

[52] T. Petrosky, I. Prigogine, and S. Tasaki, Physica A (Amsterdam) 173A, 175 (1991).

[53] S. Weinberg, Phys. Rev. 130, 776 (1963).
[54] Z. Xiao and Z.-Y. Zhou, Phys. Rev. D 94, 076006 (2016).

[55] Z. Xiao and Z.-Y. Zhou, J. Math. Phys. (N.Y.) 58, 072102 (2017).

[56] T. Sekihara, T. Hyodo, and D. Jido, Prog. Theor. Exp. Phys. 2015, $063 \mathrm{D} 04$ (2015).

[57] Z.-H. Guo and J. A. Oller, Phys. Rev. D 93, 096001 (2016).

[58] Z. Xiao and Z.-Y. Zhou, J. Math. Phys. (N.Y.) 58, 062110 (2017).

[59] U. Fano, Phys. Rev. 124, 1866 (1961).

[60] T. D. Lee, Phys. Rev. 95, 1329 (1954).

[61] P. W. Anderson, Phys. Rev. 124, 41 (1961).

[62] C. Hayne and N. Isgur, Phys. Rev. D 25, 1944 (1982).

[63] T. Barnes, N. Black, D. J. Dean, and E. S. Swanson, Phys. Rev. C 60, 045202 (1999).

[64] O. Zhang, C. Meng, and H. Q. Zheng, Phys. Lett. B 680, 453 (2009).

[65] E. S. Ackleh, T. Barnes, and E. S. Swanson, Phys. Rev. D 54, 6811 (1996).

[66] T. Barnes, N. Black, and E. S. Swanson, Phys. Rev. C 63, 025204 (2001).

[67] B. Aubert et al. (BABAR Collaboration), Phys. Rev. D 77, 011102 (2008).

[68] T. Aushev et al. (Belle Collaboration), Phys. Rev. D 81, 031103 (2010).

[69] X.-W. Kang and J. A. Oller, Eur. Phys. J. C 77, 399 (2017). 